\title{
Thermodynamic properties of fatty acid esters in some biodiesel fuels
}

\author{
A.M.Levterov ${ }^{1}$, A.A.Levterov ${ }^{2}$ \\ ${ }^{1}$ A.Podgorny Institute for mechanical engineering problems, \\ 2/10 Pozharsky St., 61046 Kharkiv, Ukraine \\ ${ }^{2}$ National University of civil protection of Ukraine, 94 Chernyshevska St., \\ 61023 Kharkiv, Ukraine
}

Received December 27, 2017

The thermophysical properties of biodiesel fuel in the high-temperature gas phase are studied for thermodynamic calculations of the working processes of piston engines. In the article the method for obtaining approximate analytical expressions of thermodynamic functions has been offered. The review of published experimental and design data enabled forming a set of thermodynamic and thermochemical properties of twenty-two complex methyl and ethyl chemically bonded esters of eleven higher fatty acids in biodiesel fuels. Next, the problem of polynomial approximation of thermodynamic functions has been solved. The coefficients of the approximating polynomials of the thermodynamic properties of esters in the gas phase have been tabulated.

Keywords: biodiesel fuel, fatty acid esters, burn process, properties, approximation, modeling.

Исследованы теплофизические свойства биодизельных топлив в высокотемпературной газовой фазе, используемые для термодинамических расчетов рабочих процессов поршневых двигателей. Предложен способ получения приближенных аналитических выражений термодинамических функций. В результате анализа опубликованных әкспериментальных и расчетных данных сформирован набор термодинамических и термохимических свойств 22 сложных метиловых и этиловых әфиров 11 высших жирных кислот, химически связанных в биодизельных топливах. Решена задача аппроксимации термодинамических функций полиномами. Коэффициенты аппроксимирующих полиномов термодинамических свойств сложных әфиров в газовой фазе сведены в таблицу.

Термодинамічні властивості складних ефірів жирних кислот низки біодизельних палив. A.М.Лєвтєров, О.А.Лєвтєров.

Досліджено теплофізичні властивості біодизельного палива у високотемпературній газовій фазі, які використовано для термодинамічних розрахунків робочих процесів поршневих двигунів. Запропоновано спосіб отримання наближених аналітичних виразів термодинамічних функцій. За підсумками аналізу опублікованих експериментальних та розрахункових даних сформовано набір термодинамічних і термохімічних властивостей 22 складних метилових і етилових ефірів 11 вищих жирних кислот, хімічно зв'язаних у біодизельних паливах. Після цього вирішено задачу апроксимації термодинамічних функцій поліномами. Коефіцієнти апроксимуючих поліномів термодинамічних властивостей складних ефірів у газовій фазі подано у таблиці. 


\section{Introduction}

In the general balance of motor fuels, those obtained from biological materials (bio ethanol and biodiesel fuel (biodiesel)) have their own stable niche. The International Energy Agency (IEA) is predicting their production growth to $25-30 \%$ in 2050 [1]. The running of a benzene-ethanol piston engine poses no exceptional problems. An entirely different matter though is biodiesel fuel, a compound organic substance in the form of various combinations of chemically bonded esters of higher fatty acids.

The laboratory of IPMash NAS of Ukraine conducted a series of motor rig tests of biodiesel fuel obtained from various vegetable oils. The results of research are presented in $R \& D$ reports and publications. However, in developing the basic mathematical model [2, 3], numerical prognosis of the process burn indicators in diesels running on biofuels or their mixtures with petroleum ones proved to be impossible. For approximate computation of thermodynamic functions (TF), the handbook for model thermophysical properties of individual substances there are no data on biodiesel fuel in the high-temperature gas phase. There are no publically available data either. Publications [4-7] provide extensive data on the thermodynamic properties of different, mainly, inorganic substances.

Publications on mathematical modeling of burn processes in biofuel diesels in most cases are restricted to reports on the results of computation with licensed software packages or to direct thermal analysis. Thus, in [8], analysis yields the composition of marginal combustion products based on the airfuel ratio and empirical formulas of the conventional molecular composition of diesel $\left(\mathrm{C}_{15.05} \mathrm{H}_{27.94}\right)$ and biodiesel $\left(\mathrm{C}_{19.03} \mathrm{H}_{35.17} \mathrm{O}_{2}\right.$ methyl ester of rapeseed oil) fuels. Here, emissions of soot, nitrogen monoxides and solid particles are not taken into account. Investigating the kinetics of combustion of biodiesel fuel has been restricted to considering esters of fatty acids with a short chain, e.g., methyl butanoate $\mathrm{C}_{5} \mathrm{H}_{10} \mathrm{O}_{2}$ with a known kinetic mechanism of oxidation [9].

Hence, having analyzed the publications, it has been required to form first a set of thermodynamic and thermochemical properties of the ester higher fatty acids in the high-temperature gas phase, and then obtain approximate expressions for TF.

\section{Database generation. Approximate TF}

With thermodynamic analysis of burn processes in a piston engine, the basic TFs are total internal energy, total enthalpy, the entropy temperature component, the isochoric and isobaric heat capacities, the adiabatic index, and others.

An approximate analytical presentation of $\mathrm{TF}$ requires forming arrays of standard formation enthalpies $\left(\Delta H_{f T}^{0}\right)$, standard temperature entropy $\left(S_{f T}\right)$, and values of entropy $(S(T))$ or enthalpy $(H(T))$ or isobaric heat capacity $\left(C_{p}(T)\right)$ within the temperatures $300-3.000 \mathrm{~K}$. Knowing the approximating expression for one of the TFs enables obtaining others by using simple thermodynamic relations.

The properties of esters are defined by the respective fatty acid [10-12]. Table 1 gives the average composition of fatty acids in some vegetable oils. In the designation of fatty acids (CX:Y) (Table 1), the first two indices show the number of carbon atoms in the fatty acid chain, and the third one, the number of olefinic links.

The information processed [13-19] helped obtain all the requisite data on the thermodynamic properties of methyl and ethyl esters of eleven higher fatty acids $\left(\Delta H_{f T}^{0}, S_{f T}, C_{p}(T), S(T), H(T)\right)$ : dodecanoic, tetradecanoic, palmitic, palmitoleic, margaric, margaroleic, stearic, oleic, linoleic, linolenic, and arachidic acids. The data presented in [13-19] have been obtained experimentally or with special analytical methods in thermal chemistry.

For the polynomial presentation of the basic esters properties, a sample of discrete values of both the specific isobaric heat ca-

Table 1. Average composition of fatty acids in some vegetable oils

\begin{tabular}{|c|c|c|c|c|c|c|c|c|c|c|c|c|c|c|c|}
\hline \multirow[b]{2}{*}{ Kind of oil } & \multicolumn{15}{|c|}{ Chain length, kind of olefinic link and mass fraction of a fatty acid, \% } \\
\hline & $\mathrm{C} 8$ & C10 & $\mathrm{C} 12$ & $\mathrm{C} 14$ & C16 & C16:1 & $\mathrm{C} 18$ & C18:1 & C18:2 & C18:3 & C20:0 & C20:1 & C22: & $\mathrm{C} 22: 1$ & C24:0 \\
\hline Soya bean oil & 0.02 & & & 0.08 & 0.61 & & 4.27 & 24.2 & 51.36 & 7.48 & 0.36 & 0.28 & 0.4 & 0.07 & 0.14 \\
\hline Palm oil & & & & & 40.6 & & 5.1 & 42.8 & 11.0 & 0.5 & & & & & \\
\hline Rapeseed oil & & & & & 4.2 & & 1.2 & 56.8 & 21.7 & 15.7 & & & & & \\
\hline
\end{tabular}


pacity $C_{p}(T)$ and entropy $S(T)$ can be used as a basic thermodynamic property [18].

Let biodiesel fuel has been presented as a set of $n$ chemically bonded esters of various fatty acids with a mass fraction $g_{i}$ each. The principle of additivity of extensive thermodynamic parameters has been used. Then, at given temperature $T$, for a thermodynamic value relating to biodiesel fuel as a whole, the following expression holds

$$
R(T)=\sum_{i=1}^{n} g_{i} r_{i}(T)
$$

where $R(T)$ is the sought for thermodynamic value; $r_{i}(T)$ is the thermodynamic value for the $i$-th fatty acid ester.

The error and the form of the polynomial that approximately describes TFs depend on the methods of fitting polynomials to tabular data. In some cases, terms have been introduced into the polynomial, which contain negative temperature exponents [5] to reduce the polynomial order whilst retaining its accuracy. The choice of the polynomial form in this paper has been defined by the basic mathematical model [2,3], its software, and the minimization therein of potential changes, which could result from introducing new data.

In the burn process model used, TF was found by using $7^{\text {th }}$-order polynomials of the form $f(T)=\sum_{i} A_{i} T^{i}$ with an initial polynomial for enthalpy [4]. The computation of TF for each ester has been based on the initial polynomial of the same form for specific isobaric heat capacity

$$
C_{p}(T)=\mathcal{F}(x)=\sum_{k=1}^{7} A_{k} \cdot x^{k}
$$

where scale factor $x=T \cdot 10^{-3} ; A_{k}$ are approximating coefficients of the approximate TF. Other required TFs are obtained by simple integration

$S_{T}=S_{T_{0}}+\int_{T_{0}}^{T} \frac{f(T)}{T} d T ; \quad H_{T}=H_{T_{0}}+\int_{T_{0}}^{T} \not{f}(T) d T$

If the initial polynomial will be the one for entropy $S_{T}=F(T)$, then enthalpy and the specific heat capacity have been computed as

$$
H_{T}=H_{T_{0}}+\int_{T_{0}}^{T} T \frac{d F(T)}{d T} d T ; \quad C_{p}=T\left(\frac{\partial S}{\partial T}\right)_{p}
$$

The integration constants were taken to be the standard substance formation enthalpy $\left(H_{T 0}=\Delta H_{f T}^{0}\right)$ and the standard temperature entropy $\left(S_{T_{0}}=S_{f T}\right)$. The values of these quantities append the table of approximating coefficients with symbols $A_{H}$ and $A_{S}$.

The real function containing the discrete $(n=10)$ values of $C_{p}(T)$ at the temperatures $300 ; 400 ; 500 ; 600 ; 800 ; 1,000 ; 1,500$; 2,$000 ; 2,500 ; 3,000 \mathrm{~K}$ is designated as $f\left(x_{i}\right)$ $=f_{i}$. The number of approximating coefficients was accepted $m=7$. Minimization of expression

$Z=\sum_{i=1}^{n}\left(f_{i}-\bar{f}\left(x_{i}\right)\right)^{2}=\sum_{i=1}^{n}\left[f_{i}-\sum_{k=0}^{m} A_{k} \cdot \bar{f}_{k}\left(x_{i}\right)\right]^{2}$

with the least-squares method yields the coefficients of approximating polynomials $A_{0}$, $A_{1}, A_{2}, \ldots, A_{m}$, which make the approximate function $\bar{f}(x)$ more specific. The approximation quality is within (0.18-0.28) and is evaluated by the mean root square deviation according to formula

$$
\delta=\left[\frac{1}{n} \cdot \sum_{i=1}^{n}\left(f\left(x_{i}\right)-f_{i}\right)^{2}\right]^{1 / 2} .
$$

The algorithm of obtaining the approximate function is in the form of a program module for changing both the form of the basic function and the system dimensionality, and obtaining the approximating function in graphic form.

The coefficients of the approximating polynomials $A_{k}$ of the thermodynamic properties of twenty-two esters of eleven higher fatty acids are shown in Table 2.

As an example, given here is the graph of the approximate dependence of isobaric heat capacity of a methyl ester of dodecanoic acid on temperature (Fig.).

The number and characteristics of oils, which can be used as raw material for biodiesel fuel, are given best in [20].

\section{Conclusions}

Solving engineering thermodynamics problems is bound to availability of information about the properties of energy carriers. In article the method for obtaining data 


\begin{tabular}{|c|c|c|c|c|c|c|c|c|c|c|c|c|c|c|c|c|c|c|c|c|c|c|c|}
\hline \multirow{3}{*}{\multicolumn{2}{|c|}{ 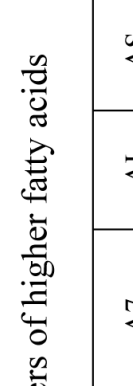 }} & 总 & î & $\stackrel{2}{8}$ & $E$ & $\underset{\infty}{\infty}$ & $\stackrel{i}{a}$ & $\begin{array}{l}n \\
2 \\
\infty \\
\infty\end{array}$ & $\stackrel{0}{\Omega}$ & $\stackrel{\circ}{\sigma}$ & $\vec{\sigma}$ & $\begin{array}{l}\stackrel{2}{f} \\
\stackrel{0}{E}\end{array}$ & $\frac{\dot{0}}{\vec{i}}$ & $\bar{\Omega}$ & $\begin{array}{l}n \\
\tilde{s} \\
\tilde{i}\end{array}$ & $\overrightarrow{\vec{i}}$ & $\begin{array}{l}0 \\
\stackrel{\text { वे }}{ }\end{array}$ & $\begin{array}{l}n \\
2 \\
2\end{array}$ & 角 & $\hat{\tilde{a}}$ & 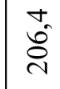 & $\underset{\vec{J}}{\bar{J}}$ & 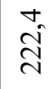 \\
\hline & & $\stackrel{g}{g}$ & $\frac{\pi}{2}$ & 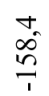 & 8 & $\frac{\infty}{0}$ & $\stackrel{0}{\frac{0}{7}}$ & $\hat{\tilde{9}}$ & $\begin{array}{l}\text { 等 } \\
\frac{1}{1}\end{array}$ & $\underset{\substack{0\\
}}{1}$ & $\begin{array}{l}\hat{\hat{\theta}} \\
\hat{a}\end{array}$ & $\begin{array}{l}n \\
\underset{j}{f} \\
\frac{f}{1}\end{array}$ & $\begin{array}{l}0 \\
0 \\
0 \\
10\end{array}$ & $\begin{array}{l}\frac{2}{2} \\
\stackrel{1}{1}\end{array}$ & 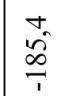 & $\begin{array}{l}\stackrel{N}{*} \\
\stackrel{y}{1}\end{array}$ & $\begin{array}{l}m \\
\hat{n} \\
n \\
1\end{array}$ & $\frac{7}{6}$ & $\begin{array}{l}a \\
\dot{d} \\
\stackrel{1}{1}\end{array}$ & $\begin{array}{l}0 \\
i \infty \\
0 \\
1\end{array}$ & $\hat{a}$ & $\begin{array}{l}\hat{0} \\
\stackrel{0}{1}\end{array}$ & 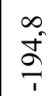 \\
\hline & \& & $\begin{array}{l}\text { to } \\
\text { Dे } \\
0 \\
0 \\
0\end{array}$ & $\begin{array}{l}\text { âे } \\
0 \\
0 \hat{\sigma} \\
\hat{\sigma}\end{array}$ & 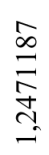 & 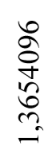 & $\begin{array}{l}\text { के } \\
0 \\
0 \\
m \\
m \\
n\end{array}$ & $\begin{array}{l}\hat{a} \\
\hat{0} \\
0 \\
\\
-\end{array}$ & 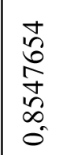 & $\begin{array}{l}\bar{n} \\
\overline{0} \\
\stackrel{0}{\circ} \\
\delta \\
\delta\end{array}$ & $\begin{array}{l}\hat{0} \\
0 \\
\text { to } \\
\text { - }\end{array}$ & 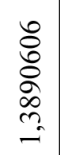 & 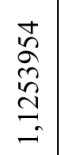 & 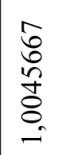 & 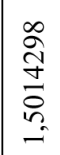 & 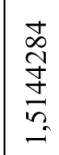 & 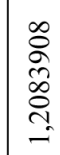 & 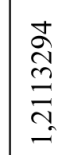 & $\begin{array}{l}\hat{2} \\
\hat{\alpha} \\
\hat{\sigma} \\
0\end{array}$ & $\begin{array}{l}\hat{0} \\
\text { ते } \\
\hat{\sigma} \\
0 \\
0\end{array}$ & 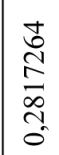 & 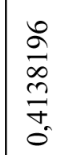 & 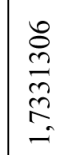 & $\begin{array}{l}\hat{f} \\
0 \\
\hat{\delta} \\
0 \\
0\end{array}$ \\
\hline $\begin{array}{l}0 \\
0 \\
0 \\
0 \\
0 \\
0 \\
2 \\
2\end{array}$ & \& & 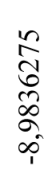 & $\begin{array}{l}\widehat{N} \\
0 \\
0 \\
0 \\
\infty \\
\sim \\
\sim \\
\infty \\
1\end{array}$ & $\begin{array}{l}\bar{\infty} \\
\hat{0} \\
\hat{\imath} \\
\hat{0} \\
1\end{array}$ & 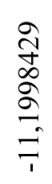 & 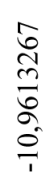 & $\begin{array}{l}0 \\
8 \\
\hat{2} \\
\infty \\
\infty \\
\infty \\
i \\
1\end{array}$ & $\begin{array}{l}\hat{a} \\
\bar{a} \\
\bar{\alpha} \\
0 \\
0 \\
i\end{array}$ & 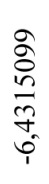 & $\begin{array}{l}\infty \\
\stackrel{2}{2} \\
\infty \\
\stackrel{2}{r} \\
\stackrel{2}{r}\end{array}$ & 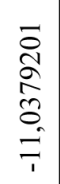 & 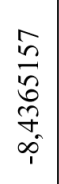 & 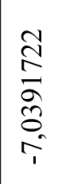 & 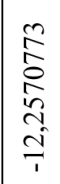 & 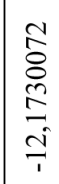 & $\mid \begin{array}{l}\vec{\sigma} \\
\infty \\
\infty \\
0 \\
0 \\
\stackrel{0}{0}\end{array}$ & 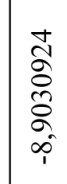 & $\begin{array}{c}2 \\
\infty \\
\infty \\
0 \\
\dot{0} \\
\dot{r} \\
i\end{array}$ & $\begin{array}{c}0 \\
\frac{0}{n} \\
\hat{o} \\
\frac{\hat{q}}{6} \\
\hat{i}\end{array}$ & $\begin{array}{l}\frac{a}{\sqrt{N}} \\
\stackrel{i}{2} \\
\frac{0}{0}\end{array}$ & $\begin{array}{l}n \\
a \\
0 \\
0 \\
0 \\
0 \\
i\end{array}$ & \begin{tabular}{l}
$\frac{a}{\Delta}$ \\
$\hat{\sigma}$ \\
$\sigma$ \\
\multirow{+}{*}{}
\end{tabular} & $\begin{array}{l}\hat{n} \\
\hat{a} \\
0 \\
m \\
m \\
m \\
1\end{array}$ \\
\hline $\begin{array}{l}\vec{E} \\
\overline{0} \\
\frac{1}{0} \\
4 \\
0 \\
0 \\
0\end{array}$ & $\&$ & 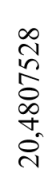 & 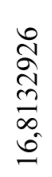 & 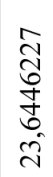 & $\begin{array}{l}0 \\
7 \\
n \\
n \\
n \\
n \\
n \\
n\end{array}$ & 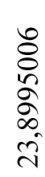 & 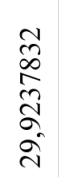 & 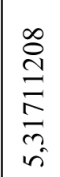 & 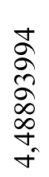 & $\begin{array}{l}\text { ते } \\
\text { છ } \\
\cdots \\
m \\
m \\
m\end{array}$ & 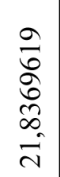 & 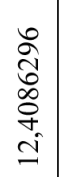 & $\begin{array}{l}n \\
0 \\
0 \\
0 \\
0 \\
0 \\
0 \\
0 \\
n\end{array}$ & 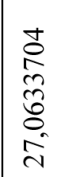 & 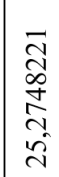 & 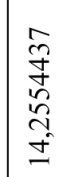 & $\begin{array}{l}0 \\
0 \\
0 \\
0 \\
0 \\
\vdots \\
\vdots \\
= \\
=\end{array}$ & $\begin{array}{l}\stackrel{2}{0} \\
\stackrel{0}{0} \\
\stackrel{2}{2} \\
\stackrel{-}{-1}\end{array}$ & 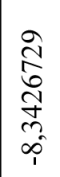 & 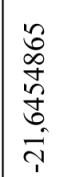 & $\begin{array}{l}0 \\
\hat{+} \\
\infty \\
\infty \\
\alpha \\
\infty \\
\frac{\infty}{1}\end{array}$ & 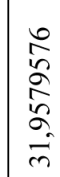 & 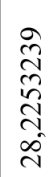 \\
\hline 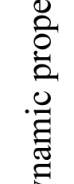 & 导 & $\begin{array}{l}2 \\
0 \\
0 \\
0 \\
0 \\
0\end{array}$ & 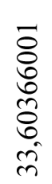 & 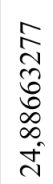 & 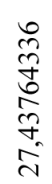 & 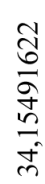 & 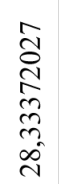 & 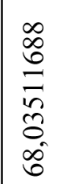 & 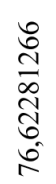 & $\begin{array}{l}2 \\
\infty \\
\infty \\
\infty \\
\infty \\
\sim \\
\stackrel{0}{+} \\
\stackrel{\sim}{\sim}\end{array}$ & 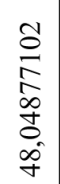 & 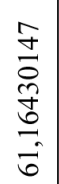 & 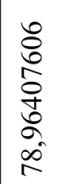 & 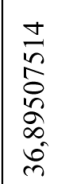 & 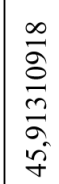 & 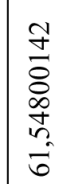 & 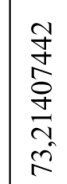 & 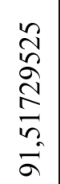 & 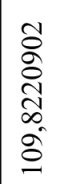 & 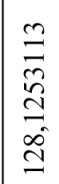 & 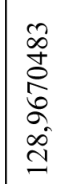 & 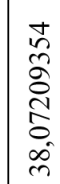 & 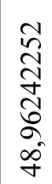 \\
\hline 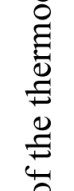 & $\underset{4}{q}$ & 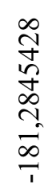 & 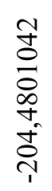 & 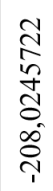 & $\begin{array}{l}0 \\
0 \\
\infty \\
0 \\
0 \\
0 \\
0\end{array}$ & $\begin{array}{l}\hat{n} \\
\widehat{\tilde{U}} \\
\tilde{\sigma} \\
\vec{\jmath}\end{array}$ & 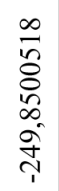 & 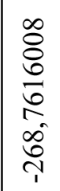 & 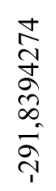 & 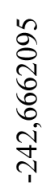 & 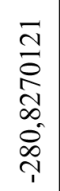 & 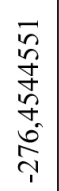 & 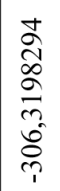 & 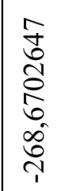 & 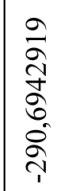 & 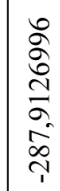 & $\begin{array}{l}m \\
\hat{y} \\
\alpha \\
\alpha \\
\frac{m}{m} \\
m\end{array}$ & 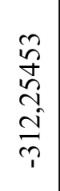 & 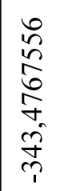 & 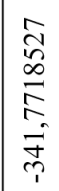 & $\begin{array}{l}0 \\
\infty \\
\infty \\
\infty \\
0 \\
0 \\
0 \\
0 \\
b \\
1 \\
1\end{array}$ & 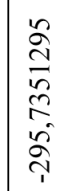 & $\begin{array}{l}\frac{1}{0} \\
\frac{\sigma}{8} \\
\frac{\delta}{\delta} \\
\frac{5}{\sigma}\end{array}$ \\
\hline $\begin{array}{l}. \frac{\pi}{E} \\
\frac{1}{0} \\
\frac{2}{0} \\
\vdots\end{array}$ & 잔 & $\begin{array}{c}n \\
d \\
0 \\
n \\
n \\
n\end{array}$ & 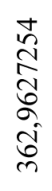 & 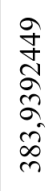 & 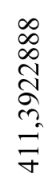 & 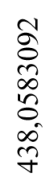 & 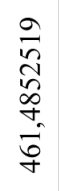 & 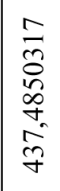 & 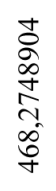 & 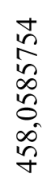 & 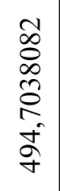 & 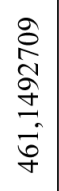 & 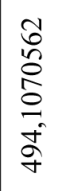 & 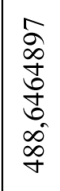 & $\begin{array}{l}\infty \\
\frac{\infty}{2} \\
\frac{2}{2} \\
\infty \\
\frac{\infty}{n}\end{array}$ & 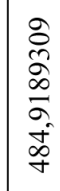 & 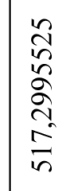 & 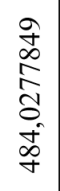 & $\begin{array}{l}2 \\
2 \\
2 \\
2 \\
0 \\
0 \\
\infty \\
i n \\
i n\end{array}$ & $\begin{array}{l}\infty \\
\infty \\
\infty \\
+ \\
\infty \\
\infty \\
\infty \\
+ \\
+ \\
+\infty\end{array}$ & 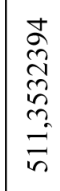 & 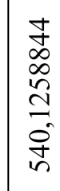 & $\begin{array}{l} \pm \\
0 \\
\infty \\
0 \\
0 \\
0 \\
\infty \\
\infty \\
0 \\
0 \\
1\end{array}$ \\
\hline 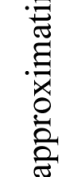 & 飞 & 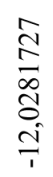 & 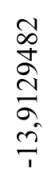 & 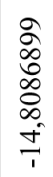 & 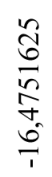 & $\begin{array}{l}\frac{\infty}{0} \\
\stackrel{0}{2}\end{array}$ & $\begin{array}{l}\hat{n} \\
\hat{2} \\
\stackrel{a}{a} \\
\stackrel{2}{a}\end{array}$ & 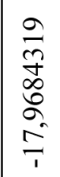 & 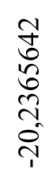 & $\begin{array}{l}\stackrel{2}{\infty} \\
\stackrel{2}{+} \\
\stackrel{2}{\gtrless} \\
\frac{\infty}{1}\end{array}$ & 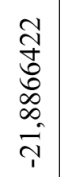 & $\begin{array}{l}\frac{\infty}{1} \\
\frac{0}{0} \\
\frac{0}{1} \\
\frac{0}{1}\end{array}$ & 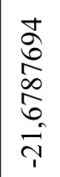 & 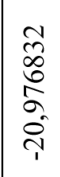 & 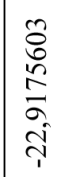 & $\begin{array}{l}\hat{n} \\
\frac{1}{5} \\
\frac{1}{2} \\
\frac{\oplus}{1} \\
i\end{array}$ & 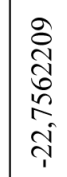 & 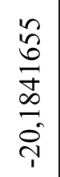 & 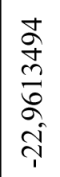 & 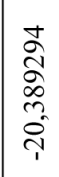 & $\begin{array}{l}\text { oे } \\
0 \\
\hat{0} \\
\infty \\
\infty \\
\vec{\imath} \\
\hat{1}\end{array}$ & 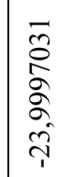 & 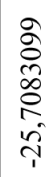 \\
\hline$\stackrel{\vec{v}}{0}$ & $\begin{array}{l}\frac{\omega}{\Phi} \\
\frac{\omega}{\omega} \\
\vec{w}\end{array}$ & $\sum^{m}$ & 㭊 & $\sum^{N}$ & H) & $\sum_{z}^{\omega}$ & $\frac{\omega}{I I}$ & $\sum_{\Sigma}^{m}$ & 岀 & $\sum_{\Sigma}^{\mathbb{N}}$ & 画 & $\sum_{\Sigma}^{\mathbb{N}}$ & 岀 & $\sum^{m}$ & 岀 & $\frac{1}{\Sigma}$ & 湈 & $\stackrel{H}{\Sigma}$ & 岀 & $\stackrel{\omega}{\Sigma}$ & 핖 & $\sum^{\mathbb{N}}$ & $\frac{\pi}{\nu 1}$ \\
\hline $\begin{array}{l}\text { N } \\
\frac{0}{D} \\
\stackrel{\pi}{\sigma}\end{array}$ & 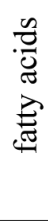 & 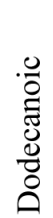 & 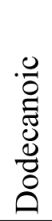 & $\cdot \frac{0}{\underbrace{2}_{n}}$ & 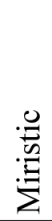 & 音 & 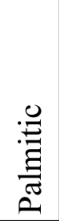 & 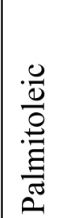 & 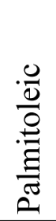 & 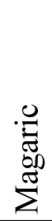 & 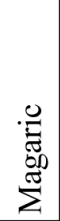 & 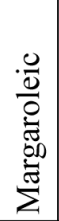 & $\begin{array}{l}\overline{\bar{\omega}} \\
\overline{0} \\
\overline{\bar{J}} \\
\overline{0} \\
\bar{\Xi}\end{array}$ & 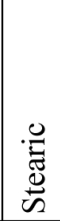 & 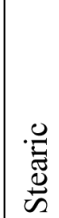 & $\frac{\frac{\pi}{2}}{0}$ & $\frac{\frac{0}{U}}{0}$ & 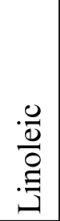 & 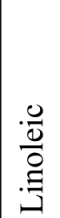 & $\frac{\mathscr{0}}{0}$ & 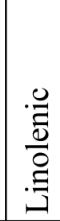 & 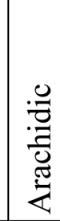 & : \\
\hline
\end{tabular}




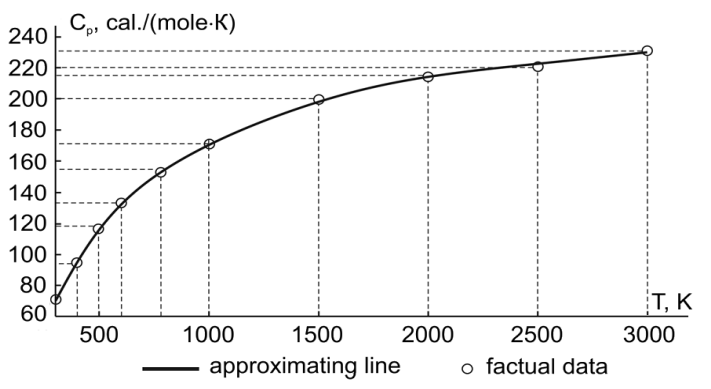

Fig.. Approximate dependence of isobaric heat capacity of a methyl ester of dodecanoic acid on temperature 21 .

on the thermophysical properties of biodiesel fuels in the high-temperature gas phase has been offered. By reviewing open-access publications, a set of data has been formed about the thermochemical and thermodynamic properties of twenty-two methyl and ethyl esters of higher fatty acids, which are chemically bonded in different combinations in biodiesel fuels. The sample obtained served for solving the problem of approximate analytical presentation of $\mathrm{TF}$ for mathematical modeling of burn processes in diesels running on both pure biodiesel fuel and bio petroleum compositions. The coefficients of approximating polynomials of the thermodynamic properties of esters of higher fatty acids have been presented in tabular form.

\section{References}

1. Technology Roadmap: Biofuels for Transport, (IEA), France (2011).

2. A.S.Kutsenko, Computer Modeling of Burn Processes in Internal Combustion Engines, Naukova Dumka, Kyiv (1988) [in Ukraine].
3. A.M.Levterov, L.I.Levterova, Visnyk NTU "KhPI", 5, 141 (2013).

4. V.Ye.Alemasov, A.F.Dregalin, Thermodynamic and Thermophysical Properties of Combustion Products, Nauka, Moscow (1971) [in Russian].

5. L.V.Gurvich et al., Thermodynamic Properties of Separate Substances, in 4 vol., Nauka, Moscow (1978-1982) [in Russian].

6. A.A.Ravdel', K.P.Mishchenko, Concise Handbook of Physical and Chemical Substances, Khimia, Leningrad (1974) [in Russian].

7. JANAF Thermochemical Tables, 2nd ed., U.S. National Bureau (1971).

8. S.Chuepeng, C.Komintarachat, J.Kasetsart, Nat.Sci., 44, 308 (2010).

9. I.G.Valeri, J.Yang, Biotechn.y Adv., 27, 641 (2009).

10. W.Yuan, A.Hansen, Q.Zhang, Fuel, 88, 1120 (2009).

11. A.K.Agarwal, Progr. Energy and Combust. Sci., 33, 233 (2007).

12. H.V.Lee, R.Yunus, J.C.Juan, Fuel Proc. Techn., 92, 2420 (2011).

13. S.P.Verevkin, Ind.Eng.Chem.Res., 48, 7388 (2009).

14. P.Larissa, R.Ceriani, R.Guirardello, Chem. Engin. Trans., 32, 535 (2013).

15. G.Toscano, D.Duca, J. Agricult. Engin., 40, 47 (2009).

16. S.Glisic, D.Skala, 9th Intern. Symp. Supercritical Fluids, France (2009, May), ISBN 978-2-9511591-7-4.

17. D.Borghi, C.Abreu, R.Guirardello, 3rd Intern. Conf. Engin. Optim., Rio de Janeiro, Brazil (2012), code paper 516 .

18. A.Osmont, L.Catoire, I.Gokalp, Intern. J. Chem.l Kinet., 39, 481 (2007).

19. A.Osmont, L.Catoire, I.Gokalp, 3rd Europ. Combustion Meeting ECM, Greece (2007), p.1.

20. E.Giakoumis, Renewable Energy, 50, 858 (2013). 